Montana Tech Library

Digital Commons @ Montana Tech

2014

\title{
Analytical Parametric Model Used to Study the Influence of Electrostatic Force on Surface Coverage During Electrospinning of Polymer Fibers
}

\author{
Joshua Beisel \\ Montana Tech of the University of Montana \\ Jerry Kyeremateng \\ Montana Tech of the University of Montana \\ Lance Purkett \\ Montana Tech of the University of Montana \\ Jessica Andriolo \\ University of Montana - Missoula \\ Jack L. Skinner \\ Montana Tech of the University of Montana
}

Follow this and additional works at: https://digitalcommons.mtech.edu/elec_engr

Part of the Nanotechnology Fabrication Commons

\section{Recommended Citation}

Beisel, J.D., Kyeremateng, J.P., L., Andriolo, J.M., \& Skinner, J.L. (2014). Analytical parametric model used to study the influence of electrostatic force on surface coverage during electrospinning of polymer fibers. Journal of Vacuum Science \& Technology B, 32, 06FI03, doi:http://dx.doi.org/10.1116/1.4900608 


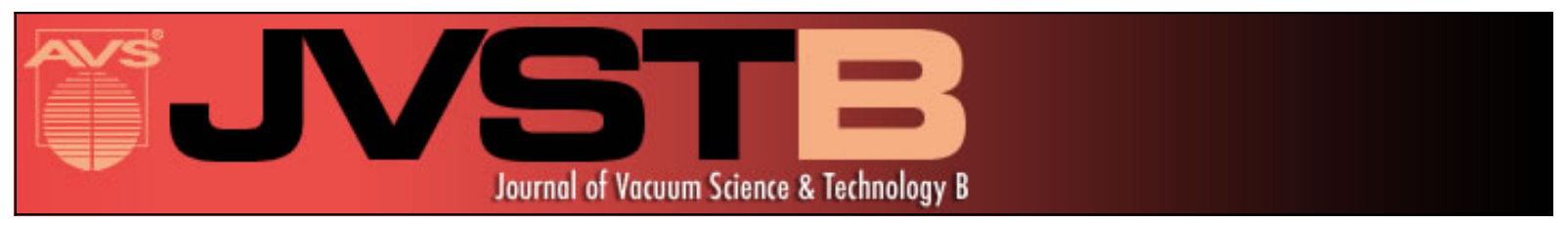

\section{Analytical parametric model used to study the influence of electrostatic force on surface coverage during electrospinning of polymer fibers}

Joshua D. Beisel, Jerry Kyeremateng, Lance Purkett, Jessica M. Andriolo, and Jack L. Skinner

Citation: Journal of Vacuum Science \& Technology B 32, 06FI03 (2014); doi: 10.1116/1.4900608

View online: http://dx.doi.org/10.1116/1.4900608

View Table of Contents: http://scitation.aip.org/content/avs/journal/jvstb/32/6?ver=pdfcov

Published by the AVS: Science \& Technology of Materials, Interfaces, and Processing

\section{Articles you may be interested in}

Fiber diameter control in electrospinning

Appl. Phys. Lett. 105, 173105 (2014); 10.1063/1.4900778

Study of polyvinyl alcohol nanofibrous membrane by electrospinning as a magnetic nanoparticle delivery approach

J. Appl. Phys. 115, 17B908 (2014); 10.1063/1.4867600

A novel mathematical model for controllable near-field electrospinning

AIP Advances 4, 017108 (2014); 10.1063/1.4861705

Modeling of solvent evaporation from polymer jets in electrospinning

Appl. Phys. Lett. 98, 223108 (2011); 10.1063/1.3585148

High performance electrospinning system for fabricating highly uniform polymer nanofibers

Rev. Sci. Instrum. 80, 026106 (2009); 10.1063/1.3079688

ionLINE

Select Si, Ge, Au and more for Advanced FIB Nanofabrication

Easy switching between multiple ion species from a single source
IONselect Technology

FIB nanofabrication beyond gallium

www.raith.com

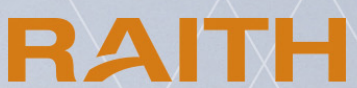

NANOFABRICATION 


\title{
Analytical parametric model used to study the influence of electrostatic force on surface coverage during electrospinning of polymer fibers
}

\author{
Joshua D. Beisel \\ Department of Electrical Engineering, Montana Tech, 1300 West Park Street, Butte, Montana 59701 \\ Jerry Kyeremateng and Lance Purkett \\ Department of General Engineering, Montana Tech, 1300 West Park Street, Butte, Montana 59701 \\ Jessica M. Andriolo \\ Bioengineering, IIP Program, University of Montana, 32 Campus Drive, Missoula, Montana 59812 \\ Jack L. Skinner ${ }^{a)}$ \\ Department of General Engineering, Montana Tech, 1300 West Park Street, Butte, Montana 59701
}

(Received 28 June 2014; accepted 17 October 2014; published 30 October 2014)

\begin{abstract}
Electrospinning (ES) can readily produce polymer fibers with cross-sectional dimensions ranging from tens of nanometers to tens of microns. Qualitative estimates of surface area coverage are rather intuitive. However, quantitative analytical and numerical methods for predicting surface coverage during ES have not been covered in sufficient depth to be applied in the design of novel materials, surfaces, and devices from ES fibers. This article presents a modeling approach to ES surface coverage where an analytical model is derived for use in quantitative prediction of surface coverage of ES fibers. The analytical model is used to predict the diameter of circular deposition areas of constant field strength and constant electrostatic force. Experimental results of polyvinyl alcohol fibers are reported and compared to numerical models to supplement the analytical model derived. The analytical model provides scientists and engineers a method for estimating surface area coverage. Both applied voltage and capillary-to-collection-plate separation are treated as independent variables for the analysis. The electric field produced by the ES process was modeled using COMSOL Multiphysics software to determine a correlation between the applied field strength and the size of the deposition area of the ES fibers. MATLAB scripts were utilized to combine the numerical COMSOL results with derived analytical equations. Experimental results reinforce the parametric trends produced via modeling and lend credibility to the use of modeling techniques for the qualitative prediction of surface area coverage from ES. (C) 2014 American Vacuum Society.
\end{abstract}

[http://dx.doi.org/10.1116/1.4900608]

\section{INTRODUCTION}

Manufacturing science and methods are central to the progression of technology. Electrospinning (ES) provides the technical community with a readily available method to produce polymer fibers ranging from nanoscale to microscale. ES produces fibers with small cross-sections and high surface area, making them ideal for a multitude of applications. Structures produced using ES methods exhibit a high surface-to-volume ratio, tunable porosity, and controllable composition. ES involves the delivery of a liquid polymer to a spinneret (or capillary) that is held at a high voltage relative to a collection plate (see Fig. 1). ${ }^{1-3}$ Fiber size depends largely on solution flow rate, supplied electric current, and fluid surface tension. ${ }^{4-6}$ ES was first observed in 1897 by Rayleigh, with related electrospraying studied in detail in 1914 and a patent issued to Antonin Formhals in 1934. ${ }^{7,8}$ In 1969, the published work of Taylor set the foundation for electrospinning. ${ }^{9}$ Although electrospinning is the common term today, it was initially described by Formhals in a series of patents as an experimental setup for the production of polymer filaments using electrostatic force. The first patent filed by Formhals in 1934 on electrospinning was issued for

${ }^{\text {a) Electronic mail: jskinner@mtech.edu }}$ the production of textile yarns, with a process consisting of a movable thread collecting device that gathered threads in a stretched condition. He was granted related patents in 1938, 1939 , and $1940 .^{10}$

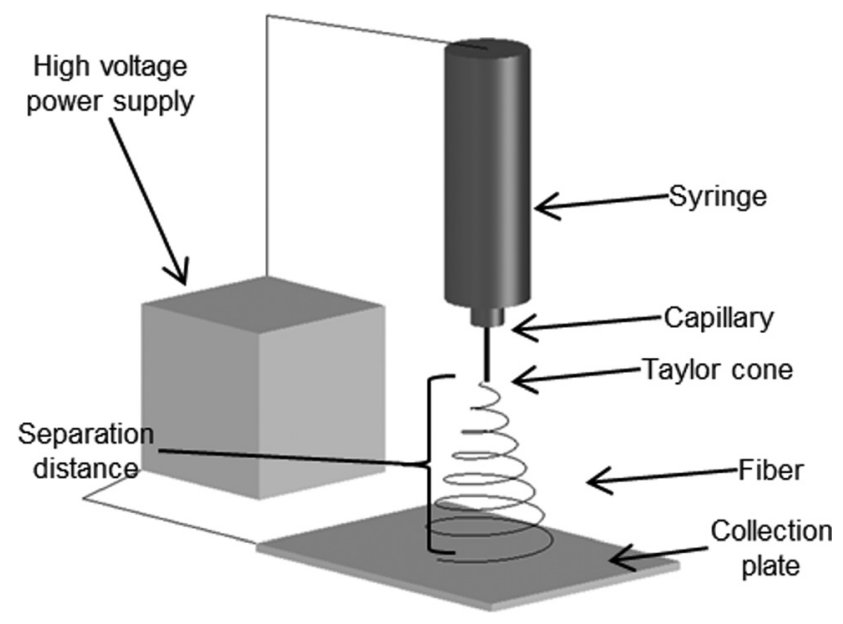

FIG. 1. Schematic of general electrospinning instrumentation. A voltage of approximately $10 \mathrm{kV}$ is applied between the capillary and the collection plate. The resultant electrostatic field causes the polar solution to be drawn out of the syringe toward the collection plate. As the fiber leaves the capillary, the solution forms a Taylor cone followed by subsequent zones of stability and instability, all within the separation distance. 
ES is initiated by electrostatic forces developed between the collection plate and the exposed surface of the polymer. Given the time scales associated with fiber deposition by ES, charges on the metallic collection plate move instantaneously. Motion of charge in the polymer (much slower than motion of charge in metals) is dictated by ionic mobility in the polymer. ${ }^{11}$ Any effort to control the electric field within ES must take into account the high-frequency cutoff enforced by polymer limitations. The low-frequency cut-off for dynamic field control relates to the spatial fiber deposition rate and time constants associated with the instability region. The short region (microns to millimeters) where the fiber is essentially straight is called the stable region. At the point where lateral perturbations cause transverse fiber velocities, the instability region starts. It is this instability region that has created so much difficulty in precise fiber control for far-field ES. ${ }^{12}$

Deitzel et al. investigated the use of a lens element to change the macroscopic electric field from the point of jet initiation to the collection target. ${ }^{13}$ Such control over the electric field was meant to dampen or eliminate the chaotic motion the material exhibits as it travels from syringe (or capillary) to collector plate, forming fibers. ${ }^{11}$ By eliminating this instability, it was suggested that better control over the deposited fibers would result. The lens element used in Deitzel's research consisted of eight copper rings, connected in series and supplied with a positive voltage, and separation distance was $20 \mathrm{~cm}$ from syringe tip to collection plate. The apparatus successfully dampened the bending instability through control of the shape and strength of the electric field present in the electrospinner. Other successes in manipulation of the electrospinning electric field have been documented. These investigations, as well as Deitzel et al.'s, take advantage of repulsive columbic forces to manipulate the flight path. The introduction of a negatively charged bar has been shown to redirect fibers, ${ }^{14}$ and electrode rods have been introduced in parallel to create an auxiliary electric field to manipulate solution flight path. ${ }^{15}$ Alternating charge between electrodes has been shown to allow aligned fiber deposition, ${ }^{16}$ and cylindrical auxiliary electrodes have been applied to multispinneret ES equipment to reduce deposition area. ${ }^{17}$

ES is of interest to the technical community in areas involving novel electrospinning methods and materials including enhanced filtration, ${ }^{18-21}$ augmented biomedical tissue regeneration, ${ }^{22,23}$ and advanced fabrication of liquid crystal polarizers. ${ }^{24}$ While a number of efforts have been attempted to provide control to far-field ES by manipulating electric fields, ${ }^{13,25-27}$ varying electrode shape, ${ }^{28}$ using rotating drums, ${ }^{12,29,30}$ and employing centrifugal methods, ${ }^{31}$ universal controllability required for broad acceptance has remained elusive.

To enable a more manufacturable process with neardeterministic deposition characteristics, the correlation between fiber deposition area, spinneret-to-collection-plate separation distance, and applied electric voltage is being studied. The use of electrospinning has expanded as fundamental knowledge of ES has increased through a combination of modeling and experimental methods. Reneker et al. provided an in-depth mathematical treatment of the bending instability in far-field ES. ${ }^{11} \mathrm{Ru}$ et al. recently published a model for controllable near-field ES. ${ }^{32}$ Nurfaizey et al. used modeling and experiment to bias the center of ES spots on collection plates. ${ }^{13,33}$ Finite element analysis (FEA) was used to verify the influence of electric field strength on spot position and aspect ratio. Heikkila et al. used FEA to relate field strength to deposition spot size. ${ }^{34}$ While FEA has use in simulation and prediction for experimental planning, an analytical model where simple parametric analysis can be readily conducted is of use to the scientist and engineer interested in ES fabrication methods. Specific focus in this article is directed at a novel derived analytical equation for predicting the deposition area covered by amorphous mats of ES fibers. The correlation between electric field intensities and the applied electrostatic forces in the vicinity of the collection plate and the deposited fiber area are compared via both modeling and experimental results.

\section{MODELING}

Model definition included a simulation sphere with a $20 \mathrm{~cm}$ diameter, designed to encompass the capillary and collection plate (see Fig. 2). The capillary was $3 \mathrm{~cm}$ in length and $1 \mathrm{~mm}$ in diameter with a $10 \mathrm{~cm}$ diameter collection plate. Dimensions of the capillary and collection plate were set to approximate the capillary and collection plate of the experimental ES instrumentation. The area within the sphere and surrounding the spinneret and collection plate were modeled as air. To assert the irrotational quality of the static electromagnetic field the charge density of the materials was assumed to be zero. Modeling assumed a steady-state condition where the field is electrostatic.

The simulation utilizes Gauss' Law of electrostatics given by

$$
\nabla \cdot(\varepsilon \nabla V)=0
$$

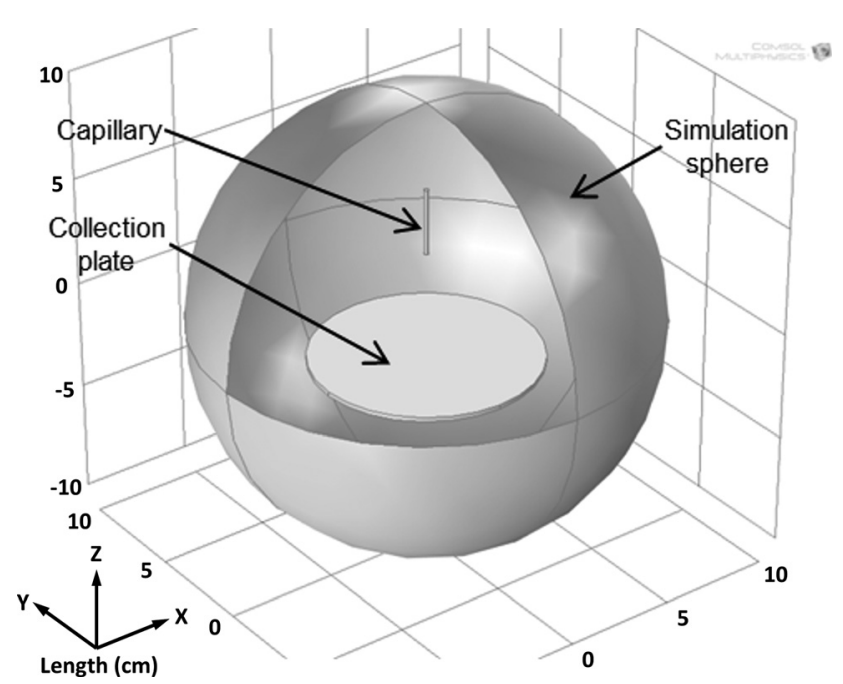

FIG. 2. Computational model of COMSOL Multiphysics simulation sphere with collection plate and capillary. The simulation sphere encompassed the spatial area of interest with an air dielectric and metallic capillary and collection plate. 


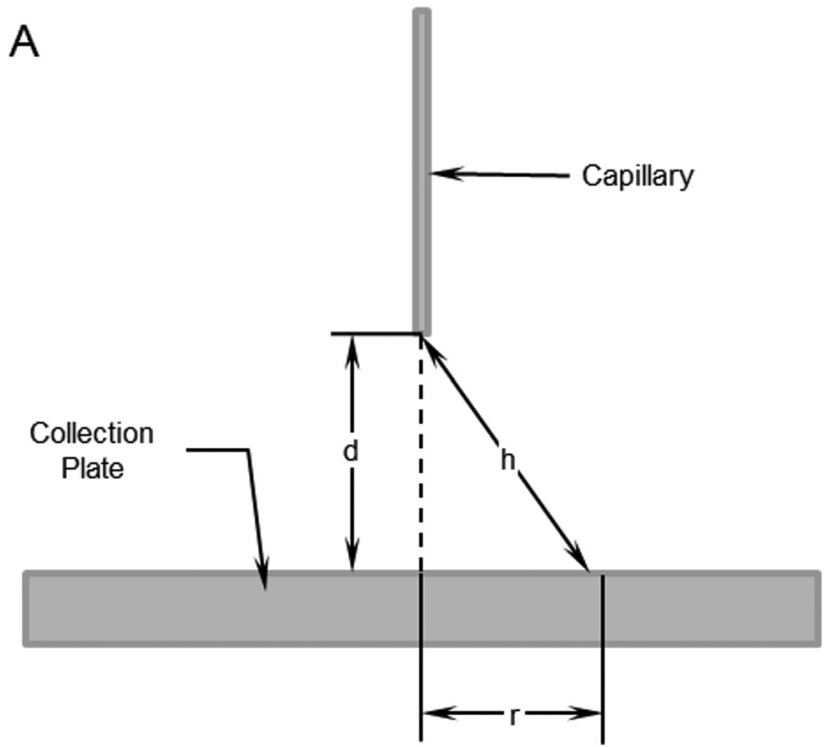

B

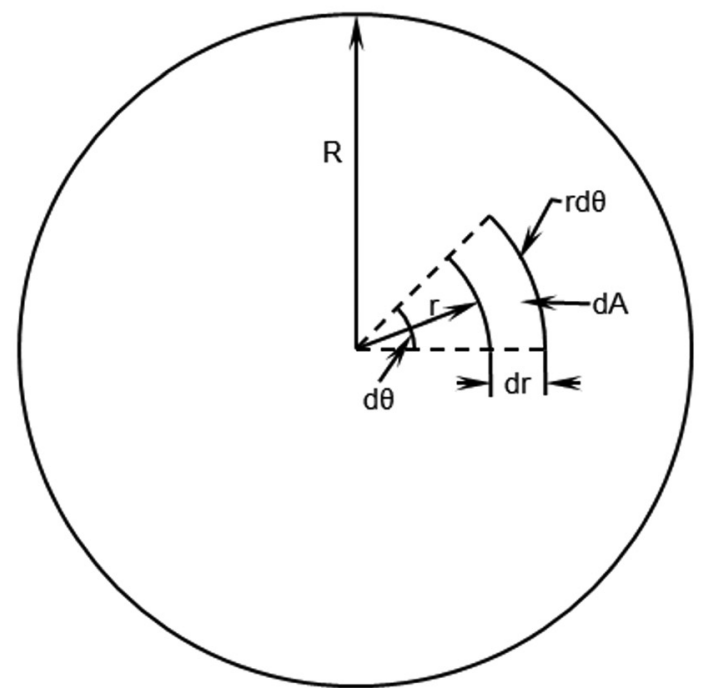

FIG. 3. (a) Schematic showing capillary and collection plate with model parameters of separation distance $(d)$ in-plane radius $(r)$ and hypotenuse $(h)$. (b) Diagram showing the differential area $(d A)$ defined in terms of radius $(r)$, differential radius $(d r)$, and differential angle $(d \theta)$.

$$
\varepsilon=\varepsilon_{\mathrm{r}} \varepsilon_{0}
$$

where $\varepsilon$ is the permittivity of the medium, $\varepsilon_{0}$ (permittivity of free space) $=8.85 \times 10^{-12} \mathrm{~F} / \mathrm{m}, \varepsilon_{\mathrm{r}}$ (relative permittivity of air) $=1$, with a dielectric constant equal to $1.0, V$ is the potential, and $\nabla$. is the divergence operator. With a focus on the effect of changes in the applied electric field, the separation distance between the capillary and the collection plate was maintained constant at $4.0 \mathrm{~cm}$. The applied voltage was varied from 8.0 to $15.0 \mathrm{kV}$ with a step size of $1.0 \mathrm{kV}$. When studying the effect of changes in the separation distance, the applied voltage was maintained constant at $12.0 \mathrm{kV}$. The separation distance was varied from 3.0 to $6.0 \mathrm{~cm}$ in steps of $1.0 \mathrm{~cm}$. The MATLAB script utilized the equation of electrostatic force to calculate the force over the collection plate. The electrostatic force was calculated using

$$
F_{e s}=\frac{\varepsilon_{r} \varepsilon_{o}}{2 d^{2}} V^{2} A
$$

where $A$ is the area of the collection plate, $V$ is the applied voltage, and $d$ is the separation distance between the capillary and the collection plate. Since the force varies with the area of the collection plate (see Fig. 3), the differential force with respect to area was calculated using

$$
d F_{e s}=\frac{\varepsilon_{r} \varepsilon_{o}}{2 d^{2}} V^{2} d A
$$

where

$$
\begin{aligned}
& d A=r d r d \theta \\
& V=\psi h=\psi\left(d^{2}+r^{2}\right)^{1 / 2}, \\
& h=\left(d^{2}+r^{2}\right)^{1 / 2} .
\end{aligned}
$$

Given Eq. (5) through Eq. (7), the electrostatic force becomes

$$
F_{e s}=\frac{\varepsilon_{r} \varepsilon_{o}}{2 d^{2}} \psi^{2}\left(d^{2}+r^{2}\right) A
$$

and the differential force $d F_{e s}$ becomes

$$
d F_{e s}=\frac{\varepsilon_{r} \varepsilon_{o}}{2 d^{2}} \psi^{2}\left(d^{2}+r^{2}\right) d A
$$

where $\psi$ is the electric field strength $(\mathrm{V} / \mathrm{m})$.

Integrating the differential force with respect to the differential area, with the assumption that the electric field is not a function of radius

$$
\begin{aligned}
& \int d F_{e s}=\frac{\varepsilon_{r} \varepsilon_{o}}{2 d^{2}} \int_{0}^{2 \pi} \int_{0}^{R} \psi^{2}\left(d^{2}+r^{2}\right) r d r d \theta \\
& F_{e s}=\frac{\varepsilon_{r} \varepsilon_{o} \psi^{2} R^{2} \pi}{2}\left(1+\frac{R^{2}}{2 d^{2}}\right) .
\end{aligned}
$$

However, matching the simulation data from COMSOL with a parabolic curve gives the following quadratic equation for the electrostatic field strength:

$$
\psi=a r^{2}+b r+c
$$

where $a, b$, and $c$ are defined as constants for each iteration of the changing modeling parameters. A more detailed differential equation where $\psi$ is assumed to be a function of radius is defined as

$$
\begin{aligned}
F_{e s}= & \frac{\varepsilon_{r} \varepsilon_{o}}{2} \int_{0}^{2 \pi} \int_{0}^{R} \psi^{2} r d r d \theta+\frac{\varepsilon_{r} \varepsilon_{o}}{2 d^{2}} \int_{0}^{2 \pi} \int_{0}^{R} \psi^{2} r^{3} d r d \theta \\
F_{e s}= & \frac{\varepsilon_{r} \varepsilon_{o}}{2} \int_{0}^{2 \pi} \int_{0}^{R}\left(a r^{2}+b r+c\right)^{2} r d r d \theta \\
& +\frac{\varepsilon_{r} \varepsilon_{o}}{2 d^{2}} \int_{0}^{2 \pi} \int_{0}^{R}\left(a r^{2}+b r+c\right)^{2} r^{3} d r d \theta
\end{aligned}
$$


Field symmetry was implied by the potential boundary condition. The Neumann type boundary $(\delta V / \delta n=0)$ was assigned to the symmetry plane, and the Dirichlet type $(V=0)$ boundary condition was assigned to the exterior surfaces of the simulation sphere. A static potential was applied to the surface of the capillary and the surface of the collection plate. The applied voltage potential is within the range of the potentials that were utilized during the electrospinning process. The simulation sphere was separated into a finite quantity of smaller, tetrahedral volumes. Within each volume, a polynomial approximation is calculated; regions of greater change have a denser mesh of calculation areas. The MATLAB script was used to extract the modeled field intensities that were generated at a distance of $1.0 \mathrm{~mm}$ above the collection plate. Subsequently, a best fit quadratic curve was calculated with parameters of $a, b$, and $c$ serving as fitting parameters. The quadratic curve was used to interpolate the field intensity at a radius of $1.25 \mathrm{~cm}$ from the capillary to examine the field at a constant radius, and an electrostatic force was determined at the fixed radius. The script utilized an iterative approach to solve for the radius and electrostatic field strength at a constant electrostatic force.
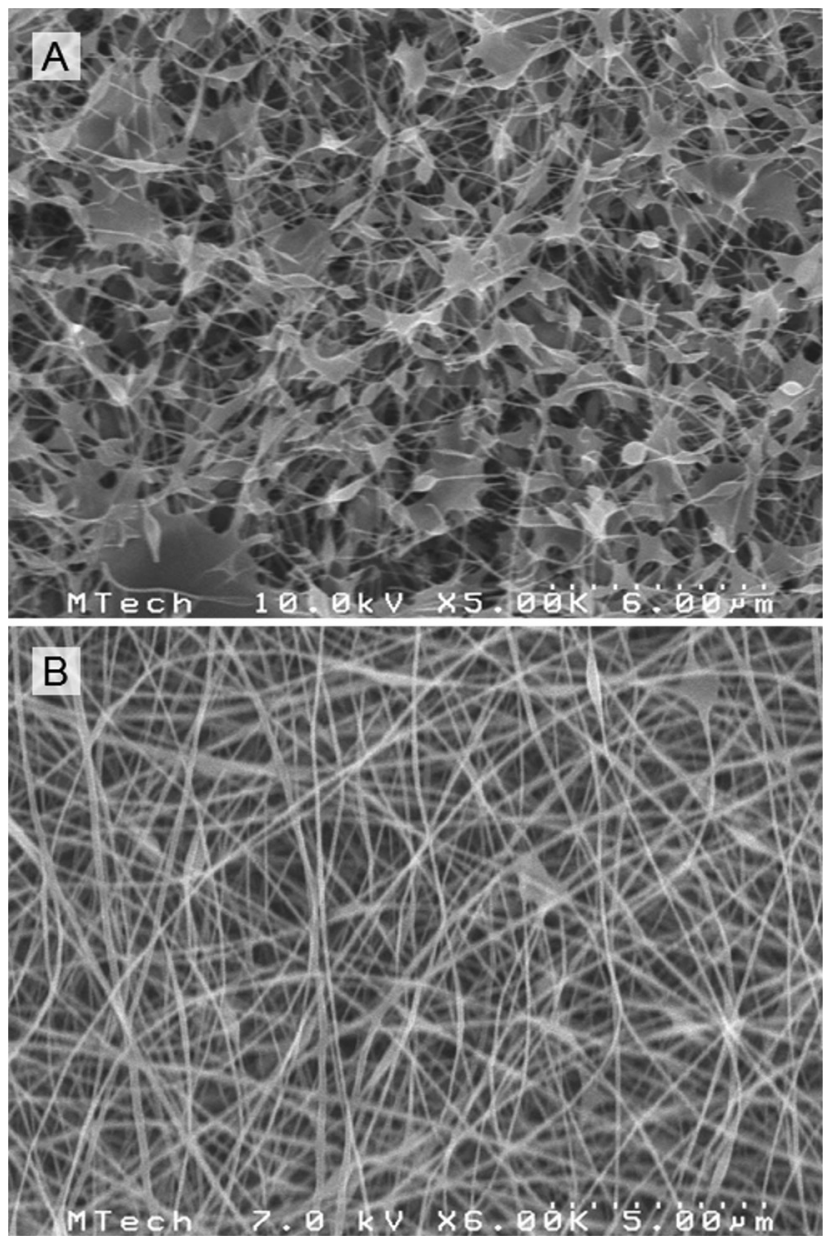

Fig. 4. SEM images of electrospun PVA fibers: (a) 4 wt. $\%, 100000 \mathrm{M}_{\mathrm{W}}$, (b) 6 wt. $\%, 100000 \mathrm{M}_{\mathrm{W}}$. Beading with a size of approximately $500 \mathrm{~nm}$ is visible in the $4 \mathrm{wt}$. \% fibers. Relatively uniform fibers are visible in the $6 \mathrm{wt}$.

$\%$ solution with fiber size ranging from 50 to $100 \mathrm{~nm}$.

\section{EXPERIMENT}

To verify the modeling results, 4 and 6 wt. \% polyvinyl alcohol (PVA) solution was electrospun according to the model parameters. As the focus of this article is the correlation between the electrostatic force and deposition spot size, 4 and 6 wt. \% PVA was used to compare the spot size of depositions that consist of highly beaded fibers and fibers with a relatively uniform cross-section (see Fig. 4). A 24 gauge capillary (outside diameter of $0.57 \mathrm{~mm}$ and inside diameter of $0.31 \mathrm{~mm}$ ) was set $4.0 \mathrm{~cm}$ above the collection plate. Samples were electrospun with voltage potentials starting at $8 \mathrm{kV}$ and increased up to $15 \mathrm{kV}$ in steps of $1 \mathrm{kV}$. The collection plate was changed for each voltage step. Electrospun fiber stability was controlled by adjusting the polymer flow rate. A fibrous mat was also deposited using a 4 wt. \% PVA solution with a constant $12.0 \mathrm{kV}$ applied voltage and an increasing separation distance starting at $3.0 \mathrm{~cm}$ and increased to $6.0 \mathrm{~cm}$ with a step size of $1.0 \mathrm{~cm}$. Fibers were deposited for a duration of approximately $2 \mathrm{~min}$ to give a deposition area visible with the naked eye on the collection plate. One sample of each PVA concentration was electrospun at each model parameter. Ten samples were electrospun using 6 wt. \% PVA with a separation distance of $4 \mathrm{~cm}$ and an applied voltage potential of $12 \mathrm{kV}$ to determine a standard deviation for the deposition spot size.

\section{RESULTS AND DISCUSSION}

Figure 5 shows an isometric view with a vertical and horizontal surface slice of the results of the COMSOL Multiphysics simulation of electrospinning with $4.0 \mathrm{~cm}$ capillary-to-collection-plate separation distance with an applied voltage at $15 \mathrm{kV}$. Figure 6 shows the electric field that surrounds the capillary on the $y-z$ plane. To eliminate some fringe effects from the corner of the collection plate the field lines were examined at $1.0 \mathrm{~mm}$ above the collection plate. The field

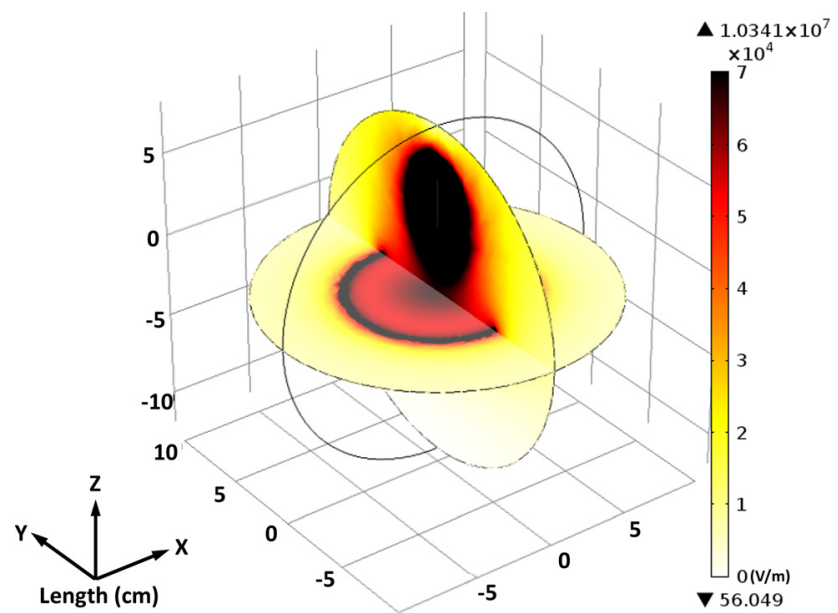

FIG. 5. (Color online) Simulated electrostatic field intensity of the spherical volume encompassing the capillary and collection plate. Field intensity varied from nearly $0 \mathrm{~V} / \mathrm{m}$ to $10 \mathrm{MV} / \mathrm{m}$, with the highest fields being neglected around the collection plate perimeter. The field strength within the central area of interest $1.0 \mathrm{~mm}$ above the collection plate varied from approximately $40 \mathrm{kV} / \mathrm{m}$ to $70 \mathrm{kV} / \mathrm{m}$. 


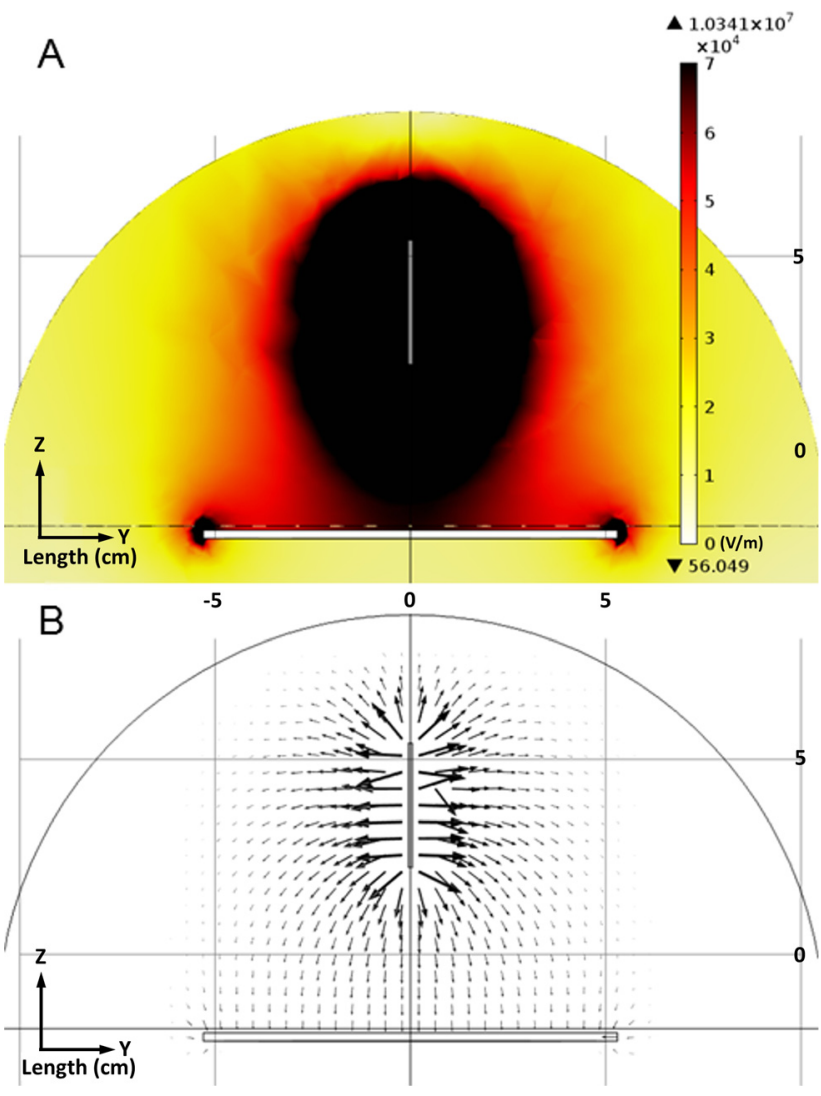

FIG. 6. (Color online) Simulated electrostatic field in a vertical surface slice through the center of the capillary and collection plate. (a) Electrostatic field strength color plot in $\mathrm{V} / \mathrm{m}$ showing the evolution of field strength in the area of interest from the needle to the collection plate. (b) Logarithmic vector plot showing the distribution of field strength from capillary to collection plate.

lines that extend from $1.0 \mathrm{~mm}$ above the collection plate to the collection plate were approximately equal. To further reduce the fringe effects at the corner of the collection plate, emphasis was given to the fields that are within a radius of
$3 \mathrm{~cm}$ from the capillary. The experimental results showed that within the voltage range of $8 \mathrm{kV}$ to $15 \mathrm{kV}$, the diameter of the lateral deposition increases up until $11 \mathrm{kV}$ and then decreases until $15 \mathrm{kV}$ (see Figs. 7 and 8). An annular shaped deposition area was apparent when the 4 wt. \% PVA, which does not allow a rapid solidification due to the increased solvent content, was electrospun (see Fig. 7). Alternatively, when a $6 \mathrm{wt}$. \% concentration, which allows rapid solidification of the fiber, was electrospun, a continuous circular deposition area was observed with an increase in visible deposition that corresponds to the inside of the annular region of the 4 wt. \% samples (see Fig. 8). At the beginning of the electrospinning process before the timed deposition, excess solution was deposited in the form of single droplets near the center of each deposition area. These droplets appear in both Figs. 7 and 8 and have negligible effect on the results of deposited spot size. Figure 9 shows a scanning electron micrograph (SEM) of the deposition area inside the annulus from Fig. 7. It can be seen that some fibers exist; however, most of the deposited material has merged. The visible annular region is due to a substantial amount of material that was deposited in this central area causing the fibers to solvent-bond into a layer that exhibited low visibility to the naked eye.

Simulation results showed that as the applied voltage increased with a constant separation distance the field strength and the electrostatic force also increased [see Fig. 10(a)]; however, the diameter of constant force decreased with a constant electrostatic force [see Fig. 10(b)]. The diameter $(2 \times$ radius $)$ of constant force is a solution to Eq. (14) where force is assumed to be a constant, and radius of the spot size becomes the variable. This is based on the premise that the area of fiber deposition can be described by a circular area where the force remains constant through varying experimental conditions. The equation was recursively solved with a MATLAB script to determine the diameter of the spot size as force was kept constant. The general trend of the

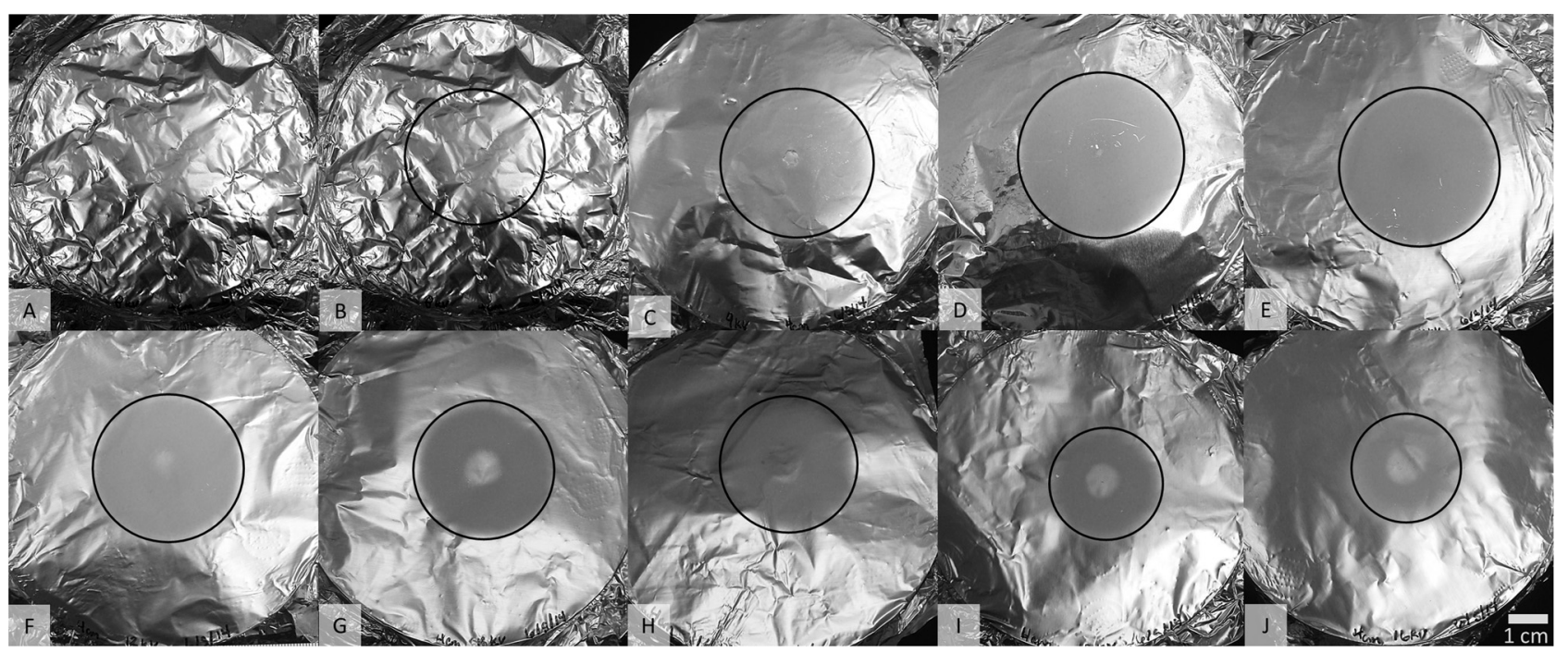

FIG. 7. Electrospun 4 wt. \% PVA deposited on aluminum foil based on matched simulation parameters with a capillary to collection plate separation distance of $4 \mathrm{~cm}$ with applied voltages at: (a) $8 \mathrm{kV}$, (b) $8 \mathrm{kV}$, (c) $9 \mathrm{kV}$, (d) $10 \mathrm{kV}$ (e) $11 \mathrm{kV}$, (f) $12 \mathrm{kV}$, (g) $13 \mathrm{kV}$, (h) $14 \mathrm{kV}$, (i) $15 \mathrm{kV}$, (j) $16 \mathrm{kV}$, with the deposition area outlined in (b)-(j). Parts (g)-(j) exhibited a visible annulus deposition area. 


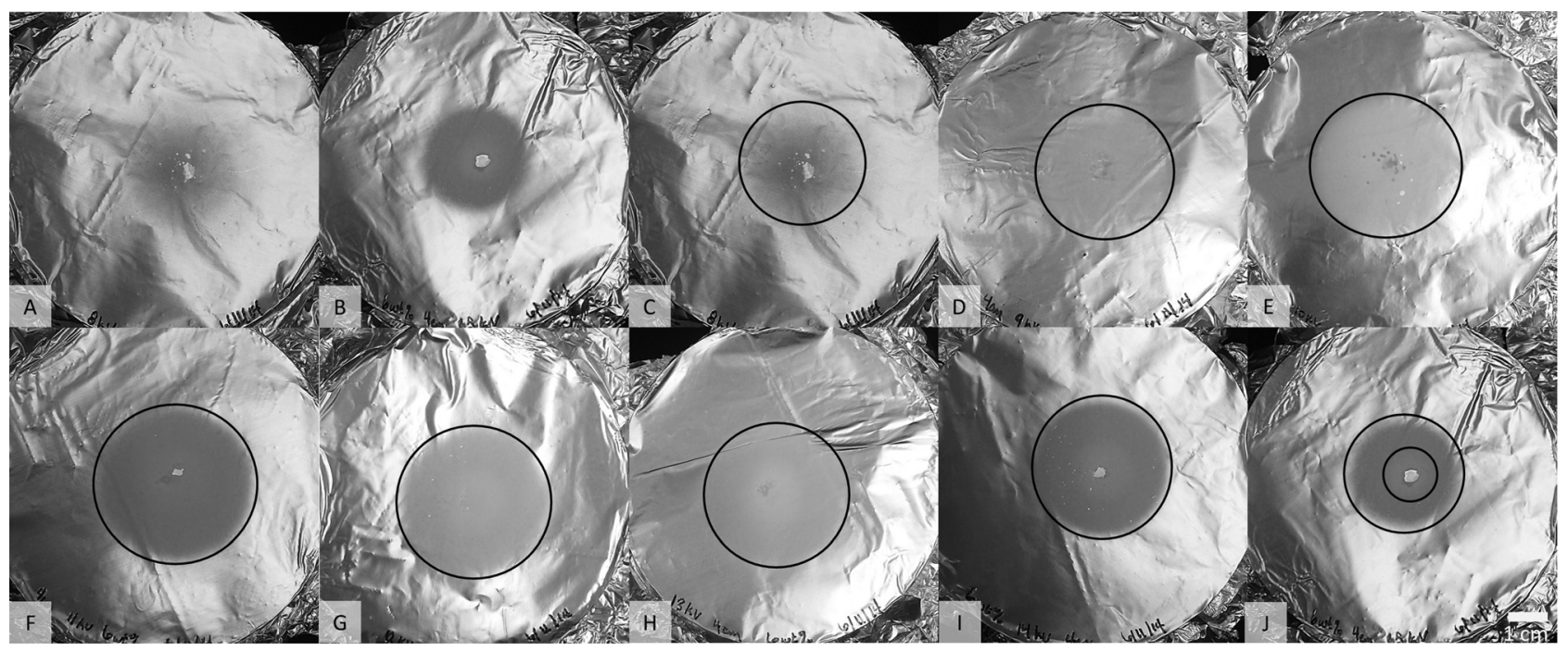

FIG. 8. Electrospun 6 wt. \% PVA deposited on aluminum foil based on matched simulation parameters with a capillary to collection plate separation distance of $4 \mathrm{~cm}$ with applied voltages at: (a) $8 \mathrm{kV}$, (b) $15 \mathrm{kV}$, (c) $8 \mathrm{kV}$, (d) $9 \mathrm{kV}$, (e) $10 \mathrm{kV}$, (f) $11 \mathrm{kV}$, (g) $12 \mathrm{kV}$, (h) $13 \mathrm{kV}$, (i) $14 \mathrm{kV}$, (j) $15 \mathrm{kV}$, with the deposition area outlined in (c)-(j) with the area of increased deposition outlined in (j).

experimental results showed an increasing deposition diameter from 8.0 to $11.0 \mathrm{kV}$ that follows the increasing trend of the electrostatic force. From 11.0 to $15.0 \mathrm{kV}$, the experimental results showed a decreasing trend similar to the decreasing diameter of the constant force. The experimental decrease in deposition diameter is qualitatively captured in the constant force diameter simulation. When the applied voltage was maintained constant and the capillary to collection plate separation distance increased, both the electrostatic field strength and the electrostatic force decrease [see Fig. 11(a)]. Both experimental deposition diameter and simulated constant force diameter increased with increasing separation distance. Discrepancies between experimental and simulated results can be accounted for in the lack of inclusion of opposing capillary forces and kinetics in the simulated results. To account for variances in experimental deposition diameter, assuming a standard normal distribution, a $99 \%$ confidence interval was determined using the

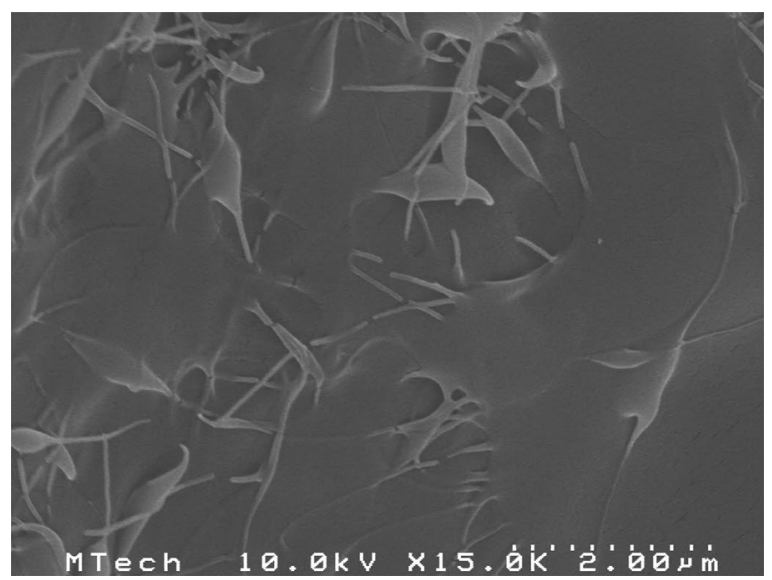

FIG. 9. SEM image of the interior of the annulus formed when electrospinning was conducted with a $14 \mathrm{kV}$ applied voltage [Fig. 7(h)]. Distinct fibers were visible; however, significant fiber merging was apparent in the central deposition area.

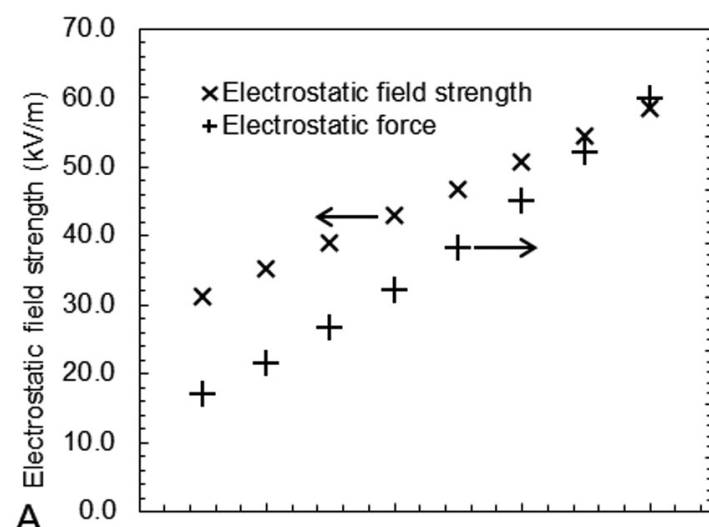

9.00

8.00

7.00

$6.00 \overline{\mathrm{z}}$

$5.00 \stackrel{8}{\circ}$

$4.00 \stackrel{\circ}{\circ}$

$3.00 \frac{\text { m. }}{\frac{\pi}{0}}$

$2.00 \frac{0}{4}$

1.00

0.00

A

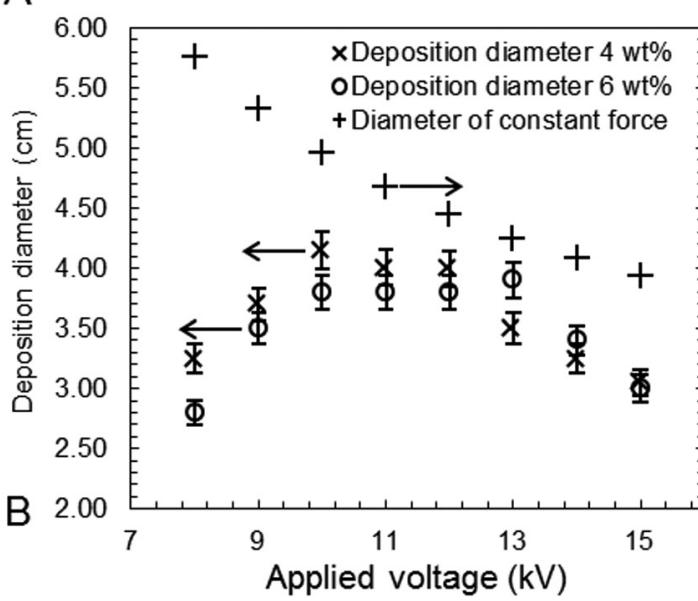

4.00

3.50

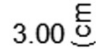

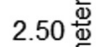

$2.00 \stackrel{\frac{\mathrm{c}}{\overline{0}}}{\frac{\mathrm{c}}{0}}$

$150 \stackrel{0}{\circ}$

$1.50 \stackrel{0}{\circ}$

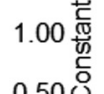

0.00

FIG. 10. Comparison of experimental and simulated results vs applied voltage. The analysis plane was $1.0 \mathrm{~mm}$ above the collection plate. (a) Electrostatic field strength $(\mathrm{kV} / \mathrm{m})$ and electrostatic force $(\mu \mathrm{N})$ vs applied voltage $(\mathrm{kV})$ for a fixed in-plane diameter of $2.5 \mathrm{~cm}$. Both electrostatic field strength and electrostatic force increase nearly linearly with the applied voltage. (b) Experimental deposition diameter (cm) for 4 and 6 wt. \% solutions and constant force diameter $(\mathrm{cm})$ vs applies applied voltage $(\mathrm{kV})$. The experimental deposition diameter increases until approximately $11 \mathrm{kV}$ and decreases through $15 \mathrm{kV}$. The experimental decrease in deposition diameter is qualitatively captured in the constant force diameter simulation. 


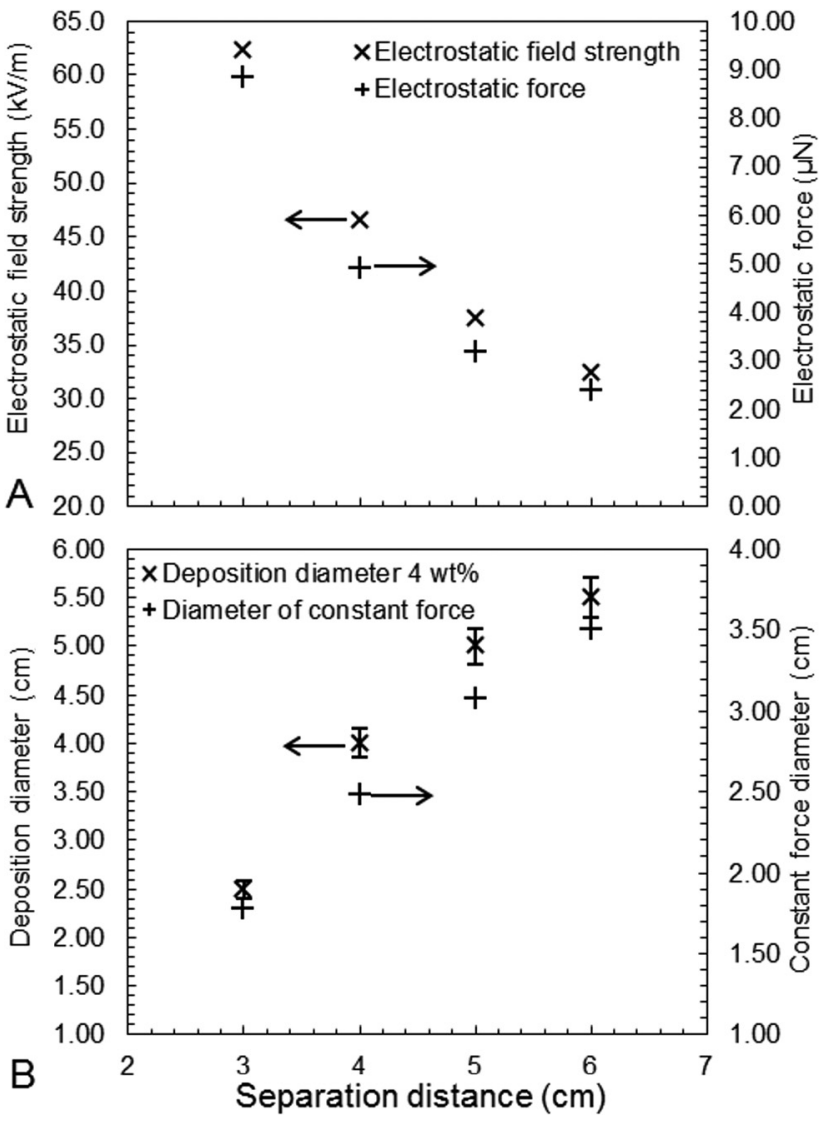

Fig. 11. Comparison of experimental and simulated results vs capillary to collection plate separation distance. The analysis plane was $1.0 \mathrm{~mm}$ above the collection plate. (a) Electrostatic field strength $(\mathrm{kV} / \mathrm{m})$ and electrostatic force $(\mu \mathrm{N})$ vs separation distance $(\mathrm{cm})$ for a fixed in-plane diameter of $2.5 \mathrm{~cm}$. Both electrostatic field strength and electrostatic force decrease with increasing separation distance. (b) Experimental deposition diameter $(\mathrm{cm})$ for the 4 wt. \% solution and constant force diameter $(\mathrm{cm})$ vs separation distance $(\mathrm{cm})$. Both experimental deposition diameter and simulated constant force diameter increase with increasing separation distance. Discrepancies between experimental and simulated results can be accounted for in the lack of inclusion of opposing capillary forces and kinetics in the simulated results.

standard deviation of the ten depositions. The $99 \%$ confidence interval was determined to be $3.79 \mathrm{~cm} \pm 0.141 \mathrm{~cm}$. The bounds of the confidence interval are $\pm 3.72 \%$ of the average deposition size. It is assumed that the other depositions will have a similar standard deviation and confidence interval.

\section{SUMMARY AND CONCLUSIONS}

This article delineates the derivation of a novel analytical equation for predicting the deposition area covered by amorphous mats of ES fibers. Experimental results were compared to modeling results with emphasis placed on the importance of electrostatic field strength and the corresponding electrostatic force on the spatial deposition of electrospun fibers. While experimental fiber deposition size was not linear throughout the experimental range of $8 \mathrm{kV}$ to $15 \mathrm{kV}$, electrostatic force plays a major role in determining deposition area, a phenomenon captured by our modeling results. An analytical expression for electrostatic force acting on fibers in the electrospinning process was derived and found to be useful for linking simulated field strength with the trends in experimental deposition diameter. The analytical model developed provides a straightforward means of performing parametric analysis of deposited fiber coverage with varying capillary-to-collection-plate separation distance and applied voltage. Discrepancies between the modeled electrostatic force and the observed experimental fiber deposition area are attributed to the dynamics involved with the fiber deposition and the opposing surface-tension forces. From $8 \mathrm{kV}$ to approximately $11 \mathrm{kV}$, the fiber deposition area increased with the electrostatic field and the electrostatic force; beyond $11 \mathrm{kV}$, the deposition area decreases, in a similar trend shown by simulation results with an area of constant electrostatic force. The results presented in this paper show qualitative agreement between many of the experimental and simulation results when considering fiber deposition area. While the results of this analysis are indicative of further consideration to create a highly accurate numerical/analytical predictive model in electrospinning, the analytical model presented has broad application in electrospinning as a parametric analysis tool.

\section{ACKNOWLEDGMENTS}

Special thanks are expressed to Sandia National Laboratories and Montana Tech for research funding and laboratory facilities. This work was partially supported by the Undergraduate Research Program and the New Faculty Seed Grant at Montana Tech.

${ }^{1}$ I. S. Chronakis, J. Mater. Process. Tech. 167, 283 (2005).

${ }^{2}$ Z. Huang, Y.-Z. Zhang, M. Kotaki, and S. Ramakrishna, Compos. Sci. Technol. 63, 2223 (2003).

${ }^{3}$ J. Doshi and D. H. Reneker, J. Electrostat. 35, 151 (1995).

${ }^{4}$ S. V. Fridrikh, J. H. Yu, M. P. Brenner, and G. C. Rutledge, Phys. Rev. Lett. 90, 144502 (2003).

${ }^{5}$ V. Beachley and X. Wen, Mat. Sci. Eng., C 29, 663 (2009).

${ }^{6}$ A. Koshi, K. Kim, and S. Shivkumar, Mater. Lett. 58, 493 (2004).

${ }^{7}$ J. Zeleny, Phys. Rev. 3, 69 (1914).

${ }^{8}$ A. Formhals, U.S. patent 1,975,504 (2 October 1934).

${ }^{9}$ G. Taylor, Proc. R. Soc. London, Ser. A 313, 453 (1969).

${ }^{10}$ K. J. Pawlowski, C. P. Barnes, E. D. Boland, G. E. Wnek, and G. L. Bowlin, MRS Proc. 827, BB1.7 (2004).

${ }^{11}$ D. H. Reneker, A. L. Yarin, H. Fong, and S. Koombhongse, J Appl. Phys. 87, 4531 (2000)

${ }^{12}$ J. A. Matthews, G. E. Wnek, D. G. Simpson, and G. L. Bowlin, Biomacromolecules 3, 232 (2002).

${ }^{13}$ J. Deitzel, J. Kleinmeyer, J. Hirvonen, and N. Beck Tan, Polymer 42, 8163 (2001).

${ }^{14}$ F. Dabirian, Y. Hosseini, and S. A. H. Ravandi, J. Text. Inst. 98, 237 (2007).

${ }^{15}$ A. H. Nurfaizey, J. Stanger, N. Tucker, N. Buunk, A. Wallace, and M. P. Staiger, J. Mater. Sci. 47, 1156 (2012).

${ }^{16}$ C. Grasl, M. Arras, M. Stoiber, H. Bergmeister, and H. Schima, Biomed. Tech. $\mathbf{5 8}(2013)$.

${ }^{17}$ G. H. Kim, Y. Cho, and W. D. Kim, Eur. Polym. J. 42, 2031 (2006).

${ }^{18}$ D. Aussawasathien, C. Teerawattananon, and A. Vongachariya, J. Membr. Sci. 315, 11 (2008)

${ }^{19}$ R. Gopal, S. Kaur, C. Y. Feng, C. Chan, S. Ramakrishna, S. Tabe, and T. Matsuura, J. Membr. Sci. 289, 210 (2007).

${ }^{20}$ K. M. Yun, C. J. Hogan, Jr., Y. Matsubayashi, M. Kawabe, F. Iskandar, and K. Okuyama, Chem. Eng. Sci. 62, 4751 (2007).

${ }^{21}$ X. Qin and S. Wang, J. Appl. Polym. Sci. 102, 1285 (2006). 
${ }^{22}$ D. Liang, B. S. Hsiao, and B. Chu, Adv. Drug Delivery Rev. 59, 1392 (2007).

${ }^{23}$ K. Kim, M. Yu, X. Zong, J. Chiu, D. Fang, Y. Seo, B. S. Hsiao, B. Chu, and M. Hadjiargyrou, Biomaterials 24, 4977 (2003).

${ }^{24}$ Y. Yao, Z. G. Gu, J. Zhang, C. Pan, Y. Zhang, and H. Wei, Adv. Mater. 19, 3707 (2007).

${ }^{25}$ P. Katta, M. Alessandro, R. Ramsier, and G. Chase, Nano Lett. 4, 2215 (2004).

${ }^{26}$ M. Acharya, G. K. Arumugam, and P. A. Heiden, Macromol. Mater. Eng. 293, 666 (2008).

${ }^{27}$ B. Sundaray, V. Subramanian, T. S. Natarajan, R. Z. Xiang, C. C. Chang, and W. S. Fann, Appl. Phys. Lett. 84, 1222 (2004).

${ }^{28}$ D. Zhang and J. Chang, Adv. Mater. 19, 3664 (2007).
${ }^{29}$ G. Liao, X. Zhou, L. Chen, X. Zeng, X. Xie, and Y. Mai, Compos. Sci. Technol. 72, 248 (2012).

${ }^{30}$ S. Y. Chew, R. Mi, A. Hoke, and K. W. Leong, Biomaterials 29, 653 (2008).

${ }^{31}$ S. Liu, Y. Long, Z. Zhang, H. Zhang, B. Sun, J. Zhang, and W. Han, J. Nanomater. 2013, 495708.

${ }^{32}$ C. Ru, J. Chen, Z. Shao, M. Pang, and J. Luo, AIP Adv. 4, 017108 (2014).

${ }^{33}$ A. H. Nurfaizey, J. Stanger, N. Tucker, N. Buunk, A. T. Wood, and M. P. Staiger, J. Eng. Fibers. Fabr. 9, 155 (2014), available at http://www.jeffjournal.org/papers/Volume9/V9I1.18.N.Tucker.pdf.

${ }^{34}$ P. Heikkilä et al., Polym. Eng. Sci. 47, 2065 (2007). 